\section{Laboratory MicroStar: A data-logging word processor for research on word processing}

\section{CHARLES F. GETTYS and LYNDON BERGLAN University of Oklahoma, Norman, Oklahoma}

Research on word processing is currently the most popular single topic in the study of human-computer interaction, in part because of Card, Moran, and Newell's (1983) powerful keystroke-level model of performance, which requires the logging of keystrokes and latencies. However, many potential researchers in this area are frustrated because of the unavailability of a data-logging word processor for the IBM PC.

Laboratory MicroStar is a word processor that logs the keystrokes and keystroke latencies of the user. It provides unobtrusive data logging of text entries, commands, and help requests for word-processing research in an IBM PC environment. The program was made completely selfdocumenting so that we could log the user's help requests for command information or general information about the program, as well as the actual commands and text entries. Laboratory MicroStar was designed to perform the routine word-processing tasks of our users, such as the writing of term papers, theses, or personal correspondence.

Laboratory Microstar was created from the program MicroStar, which is distributed as part of Borland International's Version 1.0 Editor ToolBox (Borland International, 1985). Its commands are largely a subset of the WordStar word-processing program distributed by MicroPro, and the source code is written in Version 3.0 Turbo Pascal with an occasional assembly-language subroutine. MicroStar, although it uses the same commands as does WordStar, is quite different. In the first place, it does not buffer text to disk, but instead edits memory-resident text only. Second, although it accepts WordStar commands from the keyboard, it also has a parallel system of pulldown menus, so that the user can completely bypass keyboard command entry. Finally, it is not a complete wordprocessing program as distributed by Borland; Borland's intent is that the user should modify MicroStar to add whatever additional features might be needed. For example, it is almost completely devoid of print-time formatting features, such as dot commands, double spacing, and so forth.

\section{Modifications to MicroStar}

The modifications to Borland's MicroStar consisted of more than 2,000 lines of Pascal code, and it is not possible to describe them in detail. However, we made the following major changes:

Correspondence may be addressed to Charles Gettys, Department of Psychology, University of Oklahoma, Norman, OK 73019.
Password sign-on procedure. One hazard in wordprocessing experiments is the corruption of the data of one subject by that of another subject. There are also ethical considerations that prevent associating a subject's name with his or her data. Consequently, a password routine was written. Subjects now will have to enter a password to gain access to Laboratory MicroStar, and this password has to match an encrypted password stored on the subject's text disk before the subject is allowed to proceed. The password and a total running-time stamp are entered into the logged data whenever the subject starts or ends Laboratory MicroStar.

Command help menus. The orginal MicroStar command menu system was modified into a command help system. The 57 MicroStar commands are now described in six pull-down menus, which contain logical groupings of commands. Each command is described in a short sentence or phrase. When the subject presses the dedicated f10 function key on the keyboard, this menu system is activated, and the subject can select any menu of interest. When the subject presses the letter corresponding to the command wanted on the menu, the pull-down menu is closed, and the keystroke(s) necessary to execute the command is shown for $4.4 \mathrm{sec}$ on a dedicated area of the screen. Subjects are allowed to inspect any pull-down menu before choosing a command or to exit from the menu system without choosing any command.

Screen help. Because we wanted Laboratory MicroStar to be completely self-documenting, we also added 15 help screens to teach more general concepts. These screens give word-processing tips, discuss the commands in a more general way, or discuss common difficulties that users experience with the program. The user enters the "screen help" system by pressing a second dedicated key, f9, which causes a menu describing the different screens to be displayed. Then the subject can either choose the desired screen from this menu or abort the "screen help" system.

Printing module. The primitive printing routine in Borland's MicroStar confused our pilot subjects. Consequently, this routine was rewritten to include the following features: (1) The user can now accept a default left margin or set a margin. (2) Dot commands were added for unconditional page breaks, headings, single or double spacing of text, and page numbering. An undocumented dot command was added to switch the printer to near letter quality for subjects who are writing theses or papers for publication. (3) In-text commands for underlining and boldfacing were added. (4) An ability to print any range of page numbers was added. (5) Since we had eight computers at our disposal but only two printers, we added a "print to screen" and a "print to file" feature. Therefore, subjects can now perform "pretty-printing" editing by printing to the screen, thus lessening the demands on the printer. 
Data-logging procedures. All keystrokes and latencies are logged to a RAM disk in two-byte records. This includes log-on and log-off messages, text and command entries, command help requests, and screen help requests. Considerable effort was expended to make the logged data records as compact as possible, since a typical subject types several thousand keystrokes per hour. Latencies are timed from the last keystroke, using the built-in clock in IBM PC computers, which has a nominal rate of 18.2 ticks/sec. Commands and text entries are logged by storing their ASCII values in the keystroke byte in the record. Command help requests and help screen requests (including aborts from the help systems) are logged by assigning an arbitrary code to the keystroke byte that has values greater than 127. A second byte in each record contains latency information. Data-compression techniques are used to encode latencies. Most latencies are less than 128 ticks $(7.03 \mathrm{sec})$, but occasional latencies are much longer. We encode the short latencies in one record. Latencies longer than 127 ticks are encoded in two records with the following computation for the latency byte of the first record: TRUNC (Ticks/256) OR 128; the remainder (Ticks MOD 256) is placed in the second-record latency byte. If a second record is used to encode a long latency, the keystroke field is duplicated in the second record. The advantage of this data-compression technique is that the vast majority of keystrokes is logged in one two-byte record, whereas the occasional longer latencies are accurately logged using two records. Of course, the disadvantage of this extreme data compression is that the logged records are not "human readable," and they require decoding by ancillary programs described in documentation files found on the distribution diskette(s).

\section{Limitations of MicroStar}

One irritating characteristic of MicroStar that we did not see fit to change is the way the paragraph reformat routine works. Because of the characteristics of paragraph reformatting, it is practical to enter text only in a singlespaced format with a blank line between paragraphs, even though most of our subjects want double-spaced output. We solved this problem by print-time reformatting the single-spaced text to double-spaced. It is, of course, possible to type single-spaced title pages and tables, using a dot command to suppress the print-time conversion to double spacing, but the pagination of the document is only apparent when it is printed to the screen or the printer.

Second, we installed the printer control codes directly into MicroStar. This is a limitation because the source code has to be modified for different printers.

Despite MicroStar's limitations, it has produced innumerable laboratory reports, theses, and papers for publication with few complaints from its users. It also seems to be bug-free.

\section{Supporting Software}

A number of supporting programs were written to perform chores associated with the running of a MicroStar laboratory and to perform "stand-alone" data analyses or analyses using PC-SAS and SAS on a mainframe. These programs are distributed as part of the Laboratory MicroStar package. Further details can be found in README.DOC files and in the file MICROSTA.PV2 on the diskettes.

\section{How MicroStar is Used \\ in a Laboratory Environment}

Each subject is issued two floppy disks. Disk A, which contains the MicroStar environment, is not modified by the subject. Disk B, which contains the text and datalogging files, is exclusively for the subject's use. MicroStar is loaded by inserting the floppy disks into Drives $A$ and $B$ of the computer and doing a warm or cold boot. The CONFIG.SYS file creates a RAM disk, and the AUTOEXEC.BAT file transfers the logged data file to the RAM disk and starts MicroStar. Since logged data is written to the RAM disk, data logging does not slow MicroStar, and it is completely unobtrusive to a naive subject. When the subject exits MicroStar, the AUTOEXEC.BAT file resumes control and copies the new version of the logged data file from the RAM disk back to Disk B. The subjects continue to use their text diskette until it is nearly full. At that time, the experimenter removes the logged data from the subject's text diskette to create more space.

\section{System Requirement}

To use MicroStar successfully, one should have an IBM PC computer, or a compatible clone with two $360 \mathrm{~K}$ or larger floppy disks. A hard disk is not necessary. We developed MicroStar for Zenith Model 148 computers using MS-DOS 3.1. These computers are considered to be closely compatible with IBM PCs, and the other PCcompatible computers we have tested with MicroStar ran MicroStar without errors. The computer ideally should have at least $512 \mathrm{~K} \mathrm{RAM}$, which is partitioned for the MicroStar software package into a $256 \mathrm{~K}$ user workspace and a 256K RAM disk. This allows for more than a 100K text file, which MicroStar stores on its heap in the user workspace, and up to about $200 \mathrm{~K}$ of logged data. It would be possible to operate MicroStar in a $384 \mathrm{~K}$ computer at the cost of reduced capacity for text files and logged data. Our computers have composite monochrome displays; but the program produces color codes, and it should work on either an MDA monochrome or a $25 \times 80$ color display. MicroStar also works with a Hercules Graphics card. The printer codes installed in MicroStar are for an Epson FX85 printer, set to the IBM printer mode. We have not tested these codes with other printers.

\section{Availability of the MicroStar Package}

The Laboratory MicroStar program in object-code form is hereby placed in the public domain. We cannot distribute the Pascal source-code modifications to Borland's source code except to licensed owners of Borland's Editor ToolBox in keeping with our software license agree- 
ment with Borland. We will send anybody a working copy of our Experimental MicroStar package in object-code form on request. If you send us a statement on letterhead stationary that you are a licensed owner of Borland's Toolbox, we will also send you the MicroStar modules we have modified and the source code for the utility programs. To compensate our laboratory for the cost of diskettes, mailers, and mailing, we require $\$ 10$ for the object-code diskette, which contains the entire MicroStar laboratory environment in executable form. If you want both the object- and Pascal source-code diskettes, send $\$ 25$ to Charles Gettys, Decision Processes Laboratory, Department of Psychology, University of Oklahoma, Norman,
OK 73019. Make the check payable to "Decision Processes Laboratory/Oklahoma University." A modicum of phone consulting will be provided if you obtain the source code.

\section{REFERENCES}

Borland INTERNATIONAL, INC. (1985). Turbo editor toolbox (Version 1.0) [Computer program]. Scotts Valley, CA: Author.

Card, S., Moran, T., NeWEll, A. (1983). The psychology of humancomputer interaction. Hillsdale, NJ: Erlbaum.

(Manuscript received December 14, 1988; revision accepted for publication July 14, 1989.) 